\title{
Application of digital filters in the processing of eye movement data
}

\author{
EDWARD J. ENGELKEN, KENNITH W. STEVENS, and JAMES W. WOLFE \\ Data Sciences and Clinical Sciences Divisions, United States Air Force School of Aerospace Medicine \\ Brooks Air Force Base, Texas 78235
}

\begin{abstract}
Digital filter techniques have been applied to the analysis of eye movement data. Methods were developed to calculate eye velocity and eye acceleration in real-time from an electronystagmogram (ENG) signal that was recorded using a one-pole RC high-pass filter in the preamplifier. Nonrecursive, finite impulse-response digital filters were designed to remove the effects of the RC high-pass filter and calculate the first and second time derivatives of the ENG signal, as well as remove high-frequency noise. Applying these new techniques to the analysis of vestibular nystagmus enables estimation of the transfer characteristics of the vestibuloocular system.
\end{abstract}

An accurate analysis of eye movement data is critical in studies of vestibular and oculomotor system function. A typical application is the analysis of vestibular nystagmus induced by angular acceleration or bithermal caloric stimulation, as discussed by Wolfe, Engelken, and Olson (1979) and Wolfe, Engelken, Olson, and Kos (1978). With these tests, slow-phase eye velocity is the major parameter of interest and, in the case of harmonic angular acceleration, provides the basis for study of the input/output relationships of the vestibular system. Optokinetic (OPK) nystagmus can also be evaluated; with this test, both eye velocity and eye acceleration are of interest. In OPK testing, eye velocity is compared with the stimulus field velocity and eye acceleration (which is related to the net force acting on the eye) is evaluated for symmetry in left vs. right beating nystagmus and left-eye vs. right-eye response.

Our previous approaches to the analysis of eye movements involved the use of analog computing techniques (Engelken \& Wolfe, 1977a, 1977b). We have recently developed digital methods for calculating eye velocity and eye acceleration in real-time from eye position recordings obtained during clinical vestibular evaluations. These methods are based on digital filter theory and have produced excellent results without the additional hardware required by the previous analog techniques. A well designed digital filter is more accurate than its analog counterpart and is drift free.

The purpose of this paper is not to elaborate a com-

The authors wish to thank John W. Docken, Daniel E. Dreher, and Lloyd J. Loup for their technical assistance, and Enoch G. Elliott for writing the programs used to design the digital filters. The research reported in this paper was conducted by personnel of the Data Sciences Divisions, USAF School of Aerospace Medicine, Aerospace Medical Division, AFSC, Brooks Air Force Base, Texas. Reprint requests should be sent to Edward J. Engelken, USAFSAM/BRST, Data Sciences Division, Brooks AFB, Texas 78235 . plete theory of digital filtering or to provide detailed filter design data; this information is abundantly available elsewhere (e.g., Hamming, 1977; Rabiner \& Gold, 1975). We wish only to present the methods used to design the filters employed in our present applications.

\section{RECORDING EYE MOVEMENTS}

Young and Sheena (1975) reviewed seven popular eye movement recording methods and provided an assessment of each. ${ }^{1}$ Of all the methods used, the electronystagmogram (ENG) is probably the most popular recording method used in clinical medicine; it is also widely used in research. Most researchers using ENG recordings have employed dc recordings to avoid the low-frequency distortion caused by the ac coupling often used in clinical recordings. The dc recording does avoid low-frequency distortion, but it leads to the problem of drift due to electrode polarization. The use of silver/silver-chloride electrodes has minimized this baseline drift but has not eliminated it. Recording eye movements for any period of time requires resetting the dc offset on the recorder to keep the tracing on scale.

We have eliminated the need for a dc offset adjustment control by using a precision 3 -sec time-constant one-pole RC high-pass filter in the ENG preamplifier; the balance of the recording system is dc coupled. High-quality solid state isolation amplifiers (Analog Devices, Model 283J) and instrumentation amplifiers (Analog Devices, Model 610J) are used. The effects of the RC filter are removed by including the inverse transfer function of the $\mathrm{RC}$ filter in the velocity and acceleration calculations. Digital filters can be designed to produce the time derivatives, remove high-frequency components, and approximate various transfer functions. Thus, we can use a digital filter approach to calculate eye velocity and eye acceleration, remove high. frequency noise, and correct for the distortion caused by the RC filter. 


\section{DESIGN OF BASIC DIGITAL FILTERS}

Consider an evenly spaced sequence of data values, $\mathrm{x}_{\mathrm{n}}$, digitized samples of a continuous variable, $\mathrm{x}(\mathrm{t})$, to be filtered. The data sample spacing (sampling interval) is $h$ sec. A digital filter acting on this data sequence is defined by

$$
\mathrm{y}_{\mathrm{n}}=\sum_{\mathrm{k}=-\mathrm{N}}^{\mathrm{N}} \mathrm{C}_{\mathrm{k}} \mathrm{x}_{\mathrm{n}-\mathrm{k}}+\sum_{\mathrm{k}=1}^{\mathrm{M}} \mathrm{D}_{\mathrm{k}} \mathrm{y}_{\mathrm{n}-\mathrm{k}}
$$

where $y_{n}$ represents the filter output data points, $C_{k}$ and $D_{k}$ are the filter coefficients, $N$ is the number of input values used on each side of the current input value, and $\mathrm{M}$ is the number of past output values used.

Equation 1 is the general form of a recursive digital filter. If $D_{k}=0$, then Equation 1 reduces to

$$
\mathrm{y}_{\mathrm{n}}=\underset{\mathrm{k}=-\mathrm{N}}{\mathrm{N}} \mathrm{C}_{\mathrm{k}} \mathrm{x}_{\mathrm{n}-\mathrm{k}}
$$

This nonrecursive digital filter does not utilize past values of the filter output, as does the recursive filter. An important advantage of the nonrecursive filter is that it can easily be designed to calculate the time derivative of its input. Also the nonrecursive filter has a linear phase response, making correction for phase distortion a simple matter. We have used nonrecursive filters exclusively.

Application of Equation 2 to a sequence of data points requires knowledge of input data values before and after the point of interest (i.e., to calculate the filtered data point $y_{n}$, we must have knowledge of all input data values from $x_{n-N}$ to $x_{n+N}$ ). If we filter in real-time, we have no knowledge of future data (i.e., $x_{n+1}$ to $x_{n+N}$ ). We must calculate $y_{n}$ only after we have observed the data point $x_{n+N}$. This results in a fixed time displacement (or linear phase shift) between the input data and the filtered result. This known time lag can easily be corrected for in the subsequent analysis. Data already collected and stored in the computer can be filtered directly, because all data values, past and future, are available.

In processing eye movement data, we are interested in two types of filters, bandpass (BP) and band-limited differentiating (BLD) filters. Consider the filter transfer function illustrated in Figure 1a. This is the general form of a BP filter whose gain is one in the band of interest and zero elsewhere. The frequency is normalized to the data sampling rate so that the maximum frequency (.5) represents the Nyquist frequency, or one-half the sampling rate. Setting $F_{a}$ to zero results in a lowpass (LP) filter in which $F_{b}$ is the high-frequency cutoff, and a high-pass (HP) filter is realized if $F_{b}$ is set to .5 with $F_{a}$ as the low-frequency cutoff. In this
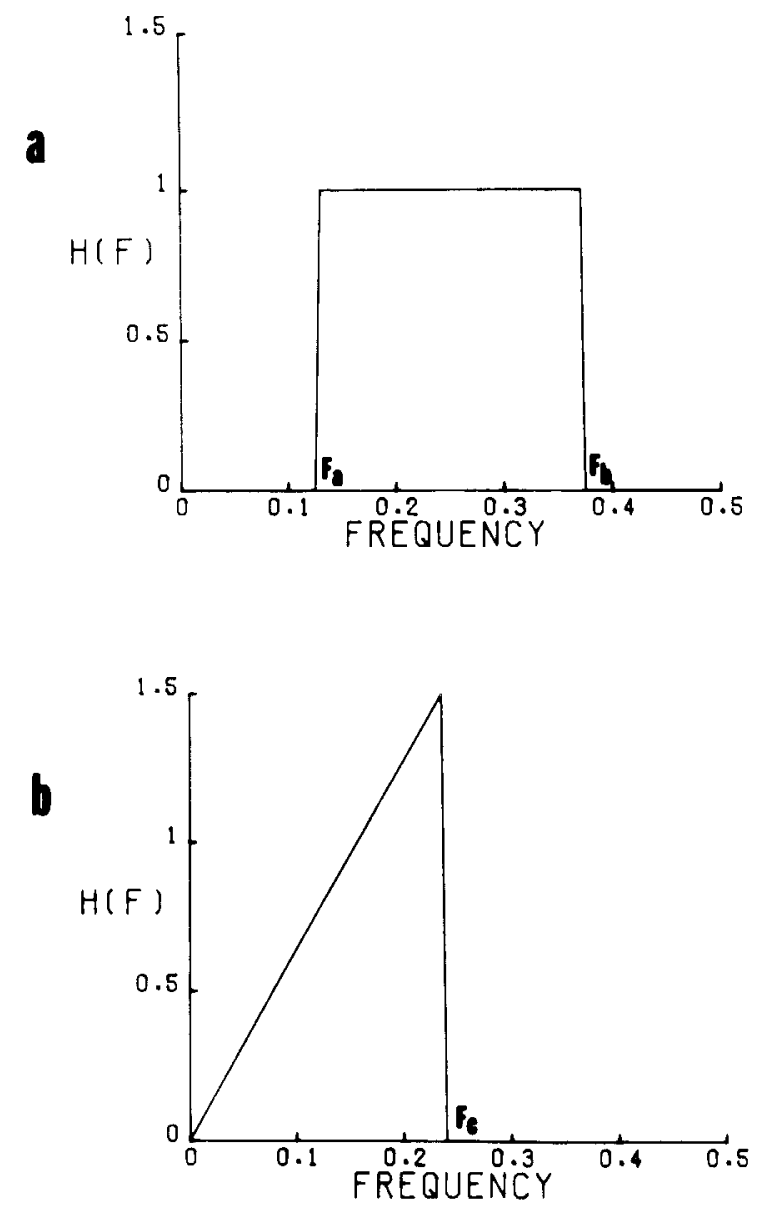

Figure 1. (a) Transfer function of an ideal bandpass filter. Gain is unity over the frequency range $F_{a}$ to $F_{b}$ and zero elsewhere. (b) Transfer function of an ideal bandlimited differentiating filter. The gain increases uniformly with a slope of $2 \pi$ up to a cutoff frequency of $F_{c}$, above which it is zero.

respect, the LP and HP filters are special cases of the BP filter.

Designing BP filters reduces to finding the filter coefficients, $C_{k}$, to be used in Equation 2. Several approaches are discussed by Hamming (1977) and Rabiner and Gold (1975). We have used the method of Hamming, in which the BP filter coefficients are obtained from a Fourier-series expansion of the ideal transfer function of Figure 1a. This is given by

$$
C_{k}=2 \int_{F_{a}}^{F_{b}} \cos 2 \pi k f d f,
$$

which reduces to

$$
C_{\mathrm{k}}=(1 / \pi \mathrm{k})\left(\sin 2 \pi \mathrm{kF}_{\mathrm{b}}-\sin 2 \pi \mathrm{kF}_{\mathrm{a}}\right)
$$

for $\mathrm{k} \neq 0$ and $\mathrm{C}_{0}=2\left(\mathrm{~F}_{\mathrm{b}}-\mathrm{F}_{\mathrm{a}}\right)$. Equation 3 has the property that $\mathrm{C}_{\mathrm{k}}=\mathrm{C}_{-\mathrm{k}}$, and thus, the filter coefficients are symmetric. 
The filter coefficients obtained from Equation 3 must be calculated for a range of $-\mathrm{N} \leqslant \mathrm{k} \leqslant \mathrm{N}$, to satisfy Equation 2. Consideration of how large $\mathrm{N}$ must be for a given level of filter performance is given by Hamming (1977). The larger $\mathrm{N}$ is, the closer the filter response will approach the ideal shape illustrated in Figure 1a. The result of truncating the filter at $N$ results in an undesirable "ringing" in the filter response (the Gibbs phenomenon) and produces a finite transition zone between the passband and stopband of the filter. The truncation effects can be controlled by using a "window" or "weighting function" to modify the filter coefficients and reduce the ringing (or ripple) at the expense of increasing the transition zone. Many such windows have been devised: the von Hann window, the Hamming window, the Kaiser window, and others. We have chosen the Kaiser window (Hamming, 1977). This is a flexible window that can be adjusted via a parameter $\alpha$ to provide whatever ripple and transition zone compromise one desires. Many of the popular windows, such as the Hamming and Blackman, are special cases of the Kaiser window. The Kaiser window weights are given by

$$
\mathrm{w}_{\mathrm{k}}=\frac{\mathrm{I}_{0}\left[\alpha \sqrt{1-(\mathrm{k} / \mathrm{N})^{2}}\right]}{\mathrm{l}_{0}(\alpha)},|\mathrm{k}| \leqslant \mathrm{N}
$$

and

$$
I_{0}(x)=1+\sum_{n=1}^{\infty}\left[(x / 2)^{n} / n !\right]^{2}
$$

where $\alpha$ is the parameter used to adjust the window shape (see Hamming, 1977). is

The resulting filter equation using the Kaiser window

$$
y_{n}=\sum_{k=-N}^{N} w_{k} C_{k} x_{n-k}
$$

The BLD filter is shown in Figure $1 b$. The transfer function is a ramp of slope $2 \pi$ up to a cutoff frequency, $F_{c}$, above which it is zero. For the transfer function in Figure 1b, the Fourier-series expansion is

$$
C_{k}^{\prime}=2 \int_{0}^{F_{c}} 2 \pi f \sin 2 \pi k f d f,
$$

which reduces to

$$
\mathrm{C}_{\mathrm{k}}^{\prime}=1 / \pi\left[\frac{\sin 2 \pi \mathrm{kF}_{\mathrm{c}}}{\mathrm{k}^{2}}-\frac{2 \pi \mathrm{F}_{\mathrm{c}} \cos 2 \pi \mathrm{kF}_{\mathrm{c}}}{\mathrm{k}}\right] .
$$

In Equation 6 we have $C_{k}^{\prime}=-C_{-k}^{\prime}$, and thus the BLD filter coefficients are antisymmetric. The BLD filter, using the Kaiser window, is also given by Equation 5, except that $(1 / \mathrm{h}) \mathrm{C}_{\mathrm{k}}^{\prime}$ replaces the $\mathrm{C}_{\mathrm{k}}$ term. The factor $(1 / h)$ is necessary to provide the proper scale factor for the derivative, since the normalized transfer function in Figure $1 \mathrm{~b}$ is based on a data sampling interval of one.

\section{COMBINING BASIC FILTERS TO BUILD COMPLEX ONES}

The LP, HP, and BP filters described above are symmetric and produce zero phase shift; the BLD filter is antisymmetric and produces a $+90-\mathrm{deg}$ phase shift. Symmetric and antisymmetric filters can be combined (by addition) to produce useful filters that are asymmetric and have phase shifts between zero and +90 deg.

\section{Calculating Eye Velocity}

Consider the problem of calculating eye velocity from an ENG signal recorded with a 3-sec time-constant RC high-pass filter in the preamplifier. The transfer function of this $R C$ filter is $G(s)=T s /(T s+1)$, where $S$ is the Laplace variable and $T$ is the RC filter time constant in seconds. The inverse of $\mathrm{G}(\mathrm{s})$ is $\mathrm{H}(\mathrm{s})=$ $(\mathrm{Ts}+1) /$ Ts. To obtain eye velocity from the ENG signal uncorrupted by the RC filter, we utilize the fact that differentiation in the time domain corresponds to multiplication by $s$ in the $s$ domain. Thus, we obtain the transfer function

$$
J(s)=s H(s)=(s+1 / T),
$$

such that

$$
y(t)=L^{-1}[(s+1 / T) X(s)],
$$

where $y(t)$ is the eye velocity derived from the ENG signal uncorrupted by the RC filter, $\mathrm{X}(\mathrm{s})$ is the Laplace transform of the ENG signal recorded using the RC filter, and $\mathrm{L}^{-1}$ is the inverse Laplace transform operator.

Equation 8 produces $y(t)$ from continuous variables in the $s$ domain. Expressing Equation 8 in terms of sampled variables and using digital filter approximations in the time domain to replace $s$ domain Laplace variables, we have

$$
y_{n}=(1 / h) \underset{k=-N}{\sum} w_{k} C_{k}^{\prime} x_{n-k}+(1 / T) \sum_{k=-N}^{N} w_{k} C_{k} x_{n-k},
$$

which reduces to

$$
\mathrm{y}_{\mathrm{n}}=\underset{\mathrm{k}=-\mathrm{N}}{\sum} \mathrm{w}_{\mathrm{k}}\left[(1 / \mathrm{h}) \mathrm{C}_{\mathrm{k}}^{\prime}+(1 / \mathrm{T}) \mathrm{C}_{\mathrm{k}}\right] \mathrm{x}_{\mathrm{n}-\mathrm{k}}
$$

where $y_{n}$ represents the eye velocity values corrected for the RC filter, $x_{n}$ represents the ENG data samples, 
$w_{k}$ represents the Kaiser window weights of Equation 4 , $h$ is the data sampling interval in seconds, $T$ is the $R C$ filter time constant in seconds, $\mathrm{C}$ represents the LP filter coefficients of Equation 3, and $\mathrm{C}_{\mathrm{k}}^{t}$ represents the BLD filter coefficients of Equation 6.

Inspection of Equation 9 shows that it is similar to Equation 5, except that the $C_{k}$ term has been replaced by $\left[(1 / h) C_{k}^{\prime}+(1 / T) C_{k}\right]$, which is a set of asymmetric filter coefficients obtained from the weighted sum of the coefficients of a BLD filter and an LP filter, each having the same $\mathbf{N}$ and cutoff frequency. The digital filter given by Equation 9 represents a band-limited, LP approximation of Equation 8 using sampled data. By using $N=15$, we have developed LP velocity calculating filters with transfer functions within $1 \%$ of the ideal transfer function of Equation 7 over the frequency range of interest.

\section{Calculating Eye Acceleration}

Eye acceleration can be calculated by first calculating velocity as described above and then passing this result through a BLD filter, producing the second derivative of the ENG signal. However, having a method of calculating acceleration directly, using a single filter, is convenient. This can be done by noting that a transfer function is needed, $K(s)=s J(s)=(s+1 / T) s$, such that

$$
z(t)=L^{-1}\{[(s+1 / T) s] X(s)\},
$$

where $z(t)$ is eye acceleration.

Equation 10 is similar to Equation 8, except for an extra $s$ that is required to provide the second time derivative. Equation 10 represents a two-stage cascade filter whose first stage is the transfer function of Equation 7 and whose second stage is simply the transfer function s. The coefficients of a single digital filter that is the result of cascading two individual filters can be calculated by convolving the coefficients of the two filters. Thus we can design a digital filter to calculate eye acceleration from the ENG signal by convolving coefficients of the filter given in Equation 9 with the coefficients of a BLD filter of the same length, $N$, and cutoff frequency. This results in a new set of filter coefficients,

$$
C_{k}^{\prime \prime}=\sum_{\ell=-2 N}^{2 N}\left[(1 / h) C_{\ell}^{\prime}+(1 / T) C_{\ell}\right] \times(1 / h) C_{k-\ell}^{\prime} .
$$

The acceleration filter is implemented by using the $\mathrm{C}_{\mathrm{k}}^{\prime \prime}$ filter coefficients in place of the $C_{k}$ coefficients in Equation 5. The convolution in Equation 11 produces a digital filter of length $4 \mathrm{~N}+1$ from the original filters of length $2 \mathrm{~N}+1$.

\section{VERIFICATION OF THE FILTER DESIGN}

Once a digital filter is designed, it is usefui to produce a Bode plot of its transfer function in order to evaluate its performance. The general form of the transfer function for a nonrecursive digital filter is $\mathrm{H}(\mathrm{f})=\mathrm{A}(\mathrm{f})+\mathrm{j} \mathrm{B}(\mathrm{f})$, where

$$
A(f)=\sum_{k=-N}^{N} w_{k} C_{k} \cos 2 \pi k f
$$

and

$$
B(f)=\sum_{k=-N}^{N} w_{k} C_{k} \sin 2 \pi k f .
$$

For the $B P$ filter, $B(f)=0$, and for a BLD filter, $A(f)=0$. However, for the asymmetric filters, the transfer function will have both real and imaginary components. The magnitude of the transfer function is given by $|H(f)|=\sqrt{\left[A(f)^{2}+B(f)\right]^{2}}$, and the phase is given by $\Phi(f)=\tan ^{-1}[B(f) / A(f)]$.

\section{RESULTS}

We have written programs in BASIC to design filters according to the methods described above, as well as to invert the resulting filters to determine the transfer functions. Figure 2 illustrates the application of velocity and acceleration calculating filters to the analysis of eye

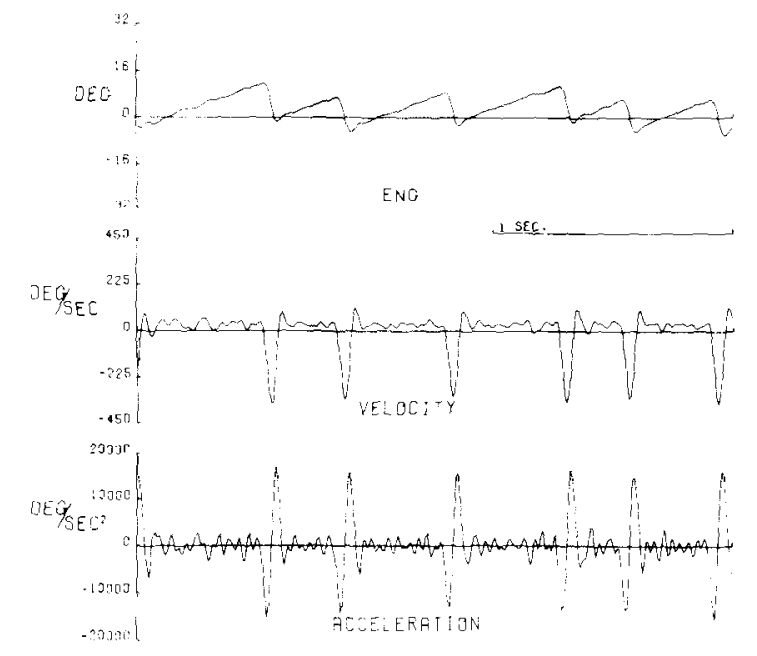

Figure 2. Application of velocity and acceleration calculating filters to the analysis of optokinetic nystagmus. Upper trace is the ENG signal sampled at $122.8 \mathrm{~Hz}$; middle trace is eye velocity; lower trace is eye acceleration. 
movements induced by constant-velocity optokinetic stimulation. Figure 3 depicts the transfer functions of the filters. The ENG signal was sampled at $122.88 \mathrm{~Hz}$; the velocity and acceleration filters had high-frequency cutoffs of $25 \mathrm{~Hz}$ and $30 \mathrm{~Hz}$, respectively.

Figure 4 shows the response of velocity and acceleration filters to vestibular nystagmus resulting from sinusoidal acceleration of a human subject at a frequency of $.08 \mathrm{~Hz}$. In this application, slow-phase velocity was the parameter of interest, and the eye acceleration and fastphase velocity data were used to identify the occurrence of fast-phase eye movements. The fast-phase velocity response was then removed from the record by linear interpolation between adjacent slow-phase velocity segments. The resulting record was ensemble averaged over four cycles of stimulation and then LP filtered using a digital filter with a $2-\mathrm{Hz}$ cutoff. The results are shown in Figure 5. Subsequent analyses of the input head velocity and resultant slow-phase eye velocity provide estimates of the gain and phase of the vestibuloocular reflex at the stimulus frequency. Also, measures of harmonic distortion and system noise may be calculated.
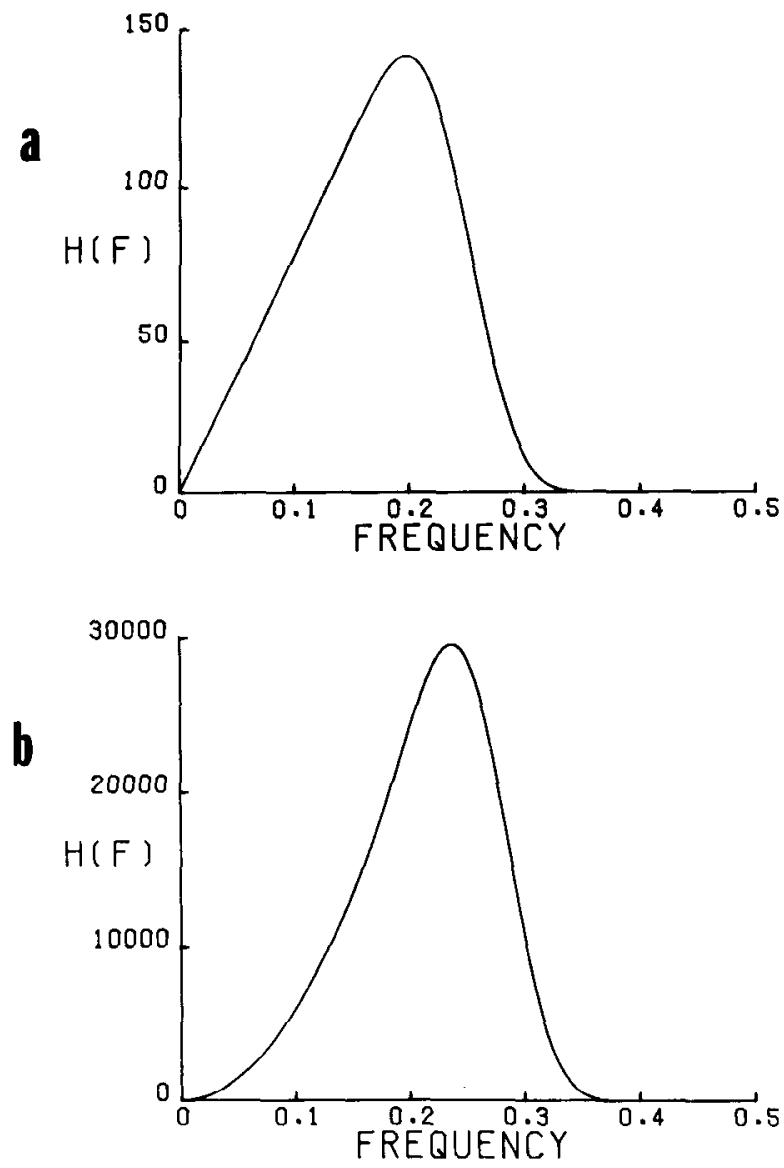

Figure 3. Magnitude of the transfer functions of the filters used to produce the responses shown in Figure 2. Upper trace is the transfer function of the velocity filter; lower trace is the acceleration filter.

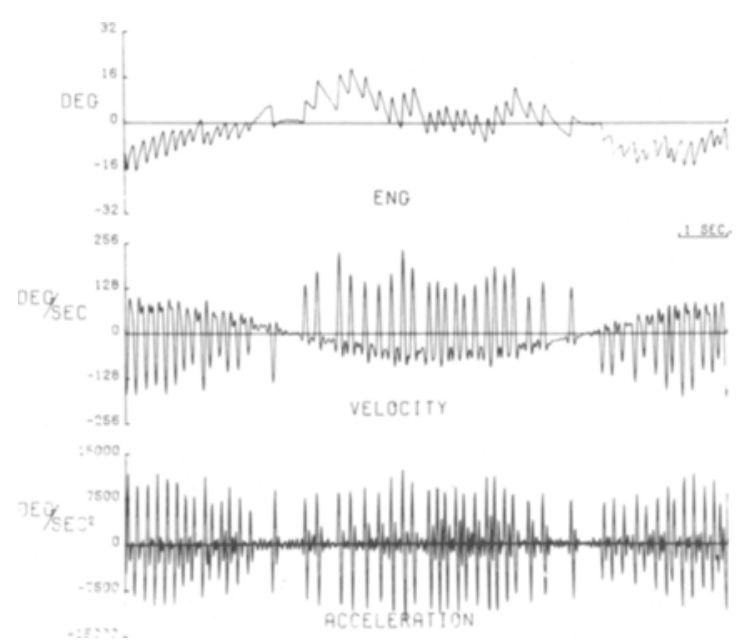

Figure 4. Response of the velocity and acceleration filters to vestibular nystagmus resulting from sinusoidal acceleration of a subject at $.08 \mathrm{~Hz}$.

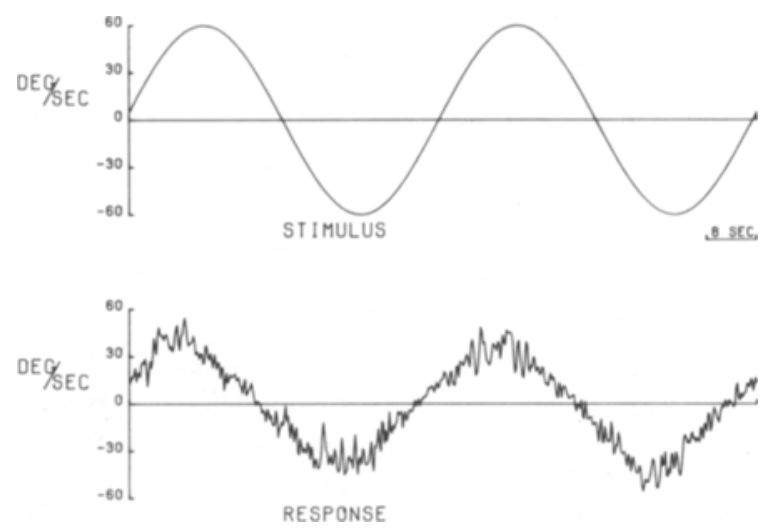

Figure 5. Head velocity (stimulus) and ensemble averaged slow phase eye velocity (response) of a human subject rotated at a frequency of $.02 \mathrm{~Hz}$,

\section{DISCUSSION}

The availability of small, economical, digital computers has made the application of digital filters to the analysis of eye movement data practical. We have replaced most of our previous analog equipment and analysis methods with digital hardware and software. We have been employing digital filter techniques for more than 1 year, using a Digital Equipment Corporation PDP-11/34 minicomputer. All filter calculations are done on-line in near real-time; only the filtered results are stored for future analysis. The use of digitalto-analog converters permits on-line monitoring of the filter outputs on our Beckman Dynograph chart recorder. Digital techniques provide stable, accurate filters whose design parameters can be altered by simply replacing one set of filter coefficients with another. The only analog filters needed are the antialiasing filters used prior to the analog-to-digital conversion of the ENG signal. 


\section{REFERENCES}

Engelken, E. J., \& Wolfe, J. W. Analog processing of vestibular nystagmus for on-line cross-correlation data analysis. Aviation, Space, and Environmental Medicine, 1977, 48, 210214. (a)

ENGELKEN, E. J., \& Wolfe, J. W. Analog signal processing of eye movements for on-line digital computer analysis. Physiology \& Behavior, 1977, 18, 157-158. (b)

Hamming, R. W. Digital filters. Englewood Cliffs, N.J: Prentice Hall, 1977.

RABiner, L. R., \& Gold, B. Theory and application of digital signal processing. Englewood Cliffs, N.J: Prentice Hall, 1975.

Wolfe, J. W., Engelken, E. J., \& Olson, J. E. Low frequency harmonic acceleration in the evaluation of surgical treatment of Meiniers' disease. Advances in Otology, Rhinology and Laryngology, 1979, 25, 192-196.

Wolfe, J. W., Engelken, E. J., Olson, J. E., \& Kos, C. M. Vestibular responses to bithermal caloric and harmonic acceler- ation. Annals of Otology, Rhinology and Laryngology, 1978, 87, 861-867.

Young, L. R., \& Sheena, D. Survey of eye movement recording methods. Behavior Research Methods \& Instrumentation, 1975, 7, 397-429.

\section{NOTE}

1. These authors also comment on the difference between electrooculography (EOG) and electronystagmography (ENG) and point out that the main difference is dc vs. ac coupling. L. B. W. Jongkees (Aspen-Snowmass Conference on Vestibular Disorders, 1979) has further emphasized that all nystagmic recording (regardless of coupling methods) should correctly be labeled ENG.

(Received for publication November 25, 1981; revision accepted February 12, 1982.) 